\title{
The interactive association between heat shock factor 1 and heat shock proteins in primary myocardial cells subjected to heat stress
}

\author{
SHU TANG ${ }^{1}$, HONGBO CHEN $^{1}$, YANFEN CHENG ${ }^{1}$, MOHAMMAD ABDEL NASIR $^{1}$, \\ NICOLE KEMPER $^{2}$ and ENDONG BAO ${ }^{1}$ \\ ${ }^{1}$ College of Veterinary Medicine, Nanjing Agricultural University, Nanjing, Jiangsu 210095, P.R. China; \\ ${ }^{2}$ Institute for Animal Hygiene, Animal Welfare and Farm Animal Behaviour, University of Veterinary \\ Medicine Hannover, Foundation, D-30559 Hannover, Germany
}

Received December 5, 2014; Accepted October 15, 2015

DOI: $10.3892 / \mathrm{ijmm} .2015 .2414$

\begin{abstract}
Heat shock factor 1 (HSF1) is a heat shock transcription factor that rapidly induces heat shock gene transcription following thermal stress. In this study, we subjected primary neonatal rat myocardial cells to heat stress in vitro to create a model system for investigating the trends in expression and association between various heat shock proteins (HSPs) and HSF1 under adverse environmental conditions. After the cells were subjected to heat stress at $42^{\circ} \mathrm{C}$ for different periods of time, HSP and HSF1 mRNA and protein levels were detected by qPCR and western blot analysis in the heat-stressed cells. The HSF1 expression levels significantly increased in the cells following 120 min of exposure to heat stess compared to the levels observed at the beginning of heat stress exposure. HSP90 followed a similar trend in expression to HSF1, whereas HSP70 followed an opposite trend. However, no significant changes were observed in the crystallin, alpha B (CRYAB, also known as HSP beta-5) expression levels during the 480-min period of exposure to heat stress. The interaction between the HSPs and HSF1 was analyzed by STRING 9.1, and it was found that HSF1 interacted with HSP90 and HSP70, and that it did not play a role in regulating CRYAB expression. Based on our findings, HSP70 may suppress HSF1 in rat myocardial cells under conditions of heat stress. Furthermore, our data demonstrate that HSF1 is not the key factor for all HSPs, and this was particularly the case for CRYAB.
\end{abstract}

\section{Introduction}

In animals, different types of stress such as heat, transportation and chemical factors contribute to lethal pathological symptoms

Correspondence to: Dr Endong Bao, College of Veterinary Medicine, Nanjing Agricultural University, Weigang 1, Nanjing, Jiangsu 210095, P.R. China

E-mail: b_endong@njau.edu.cn

Key words: heat shock proteins, heat shock factor 1, heat stress, myocardial cells, rat related to cardiovascular diseases, such as cardiac arrhythmia, seizure or hypovolemic shock with tachycardia and, eventually, circulatory collapse (1-3). In the clinical diagnosis and treatment of heat stroke, approximately $25 \%$ of patients experience failure of $\geq 1$ organ systems. In mammals, sudden death may occur as a result of stress-induced damage to cardiac tissue and myocardial cells $(4,5)$.

Heat shock proteins (HSPs) are ubiquitously expressed and highly conserved in prokaryotes and eukaryotes (6). HSP family members are molecular chaperones that are important for the regulation of several fundamental cellular processes under normal conditions (7). However, they also play a protective role during pathological processes $(8,9)$. HSPs play an important role in intracellular protein transport, cytoskeletal architecture, mutation masking, regulation of translation, intracellular redox homeostasis and protection against spontaneous or induced programmed cell death (10).

HSP70, a member of the HSP70 family, is associated with enhanced post-ischemic myocardial recovery in adult rat hearts and with the reduction of infarct size (11). It interacts with other proteins and maintains or alters their conformational states (12). Under normal conditions, heat shock factor 1 (HSF1) presents as an HSF-HSP70 heterodimer; however, HSF1 has been shown to interact with HSP70 under stress conditions (13).

HSP90, which belongs to the HSP90 family, and as previously demonstrated, does not act generally in nascent protein folding (14). At the molecular level, HSP90 binds to substrate proteins, which are in a near-native state and thus at a late stage of folding (15), poised for activation by ligand binding or interaction with other factors (16). Defects in cell physiology caused by HSP90 disruption lead to tissue- and organism-level defects. HSP90 is essential for various cellular processes, such as protein folding, protein degradation, signal transduction cascades and morphological evolution. HSP90 affinity chromatography experiments have indicated that HSP90 interacts with HSF1 in human cells (17).

HSP60, which belongs to the HSP60 family, is anti-apoptotic and provides protection against cell death by maintaining mitochondrial oxidative phosphorylation (18-20). HSP60 is typically located in the mitochondria of eukaryotic cells (21). 
It assists in the protection against protein aggregation (22) and in transporting proteins from the cytoplasm to organelles (23).

Crystallin, alpha B (CRYAB, also known as HSP beta-5) is a member of the small HSP family (24) that has chaperone-like properties, including the ability to prevent the accumulation of denatured proteins and increase cell tolerance to stress. Following its induction by cellular stresses, including heat and reactive oxygen species, CRYAB promotes cell survival and inhibits apoptosis (25). HSP induction in the myocardium may be a cardioprotective cellular response (26).

Previous studies have indicated that the dramatic increase in HSP expression is a key part of the heat shock response, which is primarily controlled by HSFs $(27,28)$. HSF1 is a major transcriptional regulator of HSPs, existing as a trimer with constitutive DNA binding activity (29). In the absence of cellular stress, HSF1 is repressed through its association with HSP. However, in response to stress, HSF1 binds to specific sequences in HSP promoters and stimulates HSP expression (30). The question as to whether HSF1 can trigger all HSPs remains unanswered. In order to address this intriguing question, in this study, we detected the levels of HSPs and HSF1 in heat-stressed rat myocardial cells in vitro and analyzed and compared the data using STRING (version 9.1) to determine the association between HSF1 and HSPs.

\section{Materials and methods}

Cell culture and exposure to heat. Primary neonatal rat myocardial cells were provided by Shanghai Fu Meng Gene Biotechnology Co., Ltd. (Shanghai, China). The cells were cultured in Dulbecco's modified Eagle's medium (DMEM, No. 11965-084) 10\%, supplemented with $10 \%$ fetal calf serum (No. 10270-098) were purchased from Gibco, Thermo Scientific, Shanghai, China, at $37^{\circ} \mathrm{C}$ in $5 \% \mathrm{CO}_{2}$ for 3 days; the viability was $>85 \%$. The cells were divided into different experimental groups, each consisting of 9 cell culture plates. Heat-stressed cells were exposed to heat at $42^{\circ} \mathrm{C}$, whereas the control cells were exposed to a normal temperature of $37^{\circ} \mathrm{C}$. One plate from each group was removed from the incubator at the start of the experiment ( $0 \mathrm{~min})$ and after 10, 20, 40, 60, 120, 240, 360 and $480 \mathrm{~min}$.

Semi-quantitative detection of HSP and HSFl expression levels by western blot analysis. The heat-stressed cells were washed with phosphate-buffered saline (PBS) 3 times, and proteins were extracted by lysis in sodium dodecyl sulfate (SDS)-polyacrylamide gel Laemmli sample buffer. The protein extracts were boiled for $5 \mathrm{~min}$ prior to loading equal amounts of protein $(10 \mu \mathrm{g})$ for $10 \%$ SDS-polyacrylamide gel electrophoresis. Proteins were transferred onto nitrocellulose membranes by electrotransfer and the membranes were blocked with 5\% skimmed milk in Tris-buffered saline $[20 \mathrm{mM}$ Tris- $\mathrm{HCl}$ (pH 7.6), $137 \mathrm{mM} \mathrm{NaCl}$ ] containing 0.1\% Tween-20 (TBST) for $1 \mathrm{~h}$ at room temperature. The membranes were incubated with anti-rat HSF1 monoclonal antibody [1:1,000; ab61382; Abcam Trading (UK) Company Ltd.], anti-rat HSP90 monoclonal antibody [ab79849; Abcam Trading (UK) Company Ltd.], anti-rat HSP70 monoclonal antibody [1:1,000; ab5442; Abcam Trading (UK) Company Ltd.], anti-rat CRYAB monoclonal antibody [1:1000; ab13496; Abcam Trading (UK) Company Ltd.], or anti-rat $\beta$-actin (ACTB) monoclonal antibody [1:1,000, ab8224;
Abcam Trading (UK) Company Ltd.] for $16 \mathrm{~h}$ at $4^{\circ} \mathrm{C}$. After washing with TBST, the membranes were incubated with peroxidase-conjugated goat anti-mouse immunoglobulin $\mathrm{G}$ at room temperature for $1 \mathrm{~h}$, and the antibody-antigen complexes were detected using Western Blotting Luminol Reagent (Santa Cruz Biotechnology, Inc., Santa Cruz, CA, USA). Bands on the developed film were quantified using Quantity One software version 4.6.2 (Bio-Rad, Hercules, CA, USA). The intensity of each band was normalized to that of $\beta$-actin.

Total RNA isolation and reverse transcription-PCR. Total RNA was isolated from the cells in the experimental and control groups using TRIzol reagent according to the manufacturer's instructions (Trizol-RNAiso Plus reagent, D9108A; Takara, China). The RNA concentrations were measured at $260 \mathrm{~nm}$ using a spectrophotometer (M200PRO; Tecan, Austria). Serial dilutions of RNA were prepared with ribonuclease-free water; $2 \mu \mathrm{g}$ of each sample were reverse transcribed using a Transcript Moloney murine leukemia virus (M-MLV) kit (Invitrogen, Shanghai, China) according to the manufacturer's instructions and stored at $-80^{\circ} \mathrm{C}$ until use. Random decamers and oligo(dT) were obtained from a RETROscript kit (AM1710; Ambion, Austin, TX, USA).

Primers. Primers were designed to anneal specifically to each target mRNA. HSF 1, HSP90, HSP70, CRYAB and $\beta$-actin mRNA sequences were obtained from the National Center for Biotechnology Information (Bethesda, MD, USA) GenBank database (accession nos: NC_005108.2, NP_077369.1 and NC_005111.2). The primers were designed using Primer Premier 5.0 software for conventional and reverse transcription-PCR amplification. The sequences were as follows: HSF1 sense, 5'-ACCCCAGCCTCTGCCTGCT-3' and antisense, 5'-TTCCCACTCGGGCTCCAGCA-3'; HSP90 sense, 5'-CCC GGTGCGGTTAGTCACGT-3' and antisense, 5'-TCCAGAGC GTCTGAGGAGTTGGA-3'; HSP70 sense, 5'-GTCCCTCA AGAGCCCAACCCCAT-3' and antisense, 5'-ACGTGGTCT AGTGGAAGCCACCA-3'; $C R Y A B$ sence, 5'-CGTCGGCTG GGATCCGGTACT-3' and antisence, 5'-CACGAAGAGCG CCAGGACGA-3'; $\beta$-actin sense, 5'-CCCATCTATGAGG GTTCA-3' and antisense, 5'-TCACGCACGATTTCC-3'. The expected lengths of the HSF 1, HSP90, HSP70, CRYAB and $\beta$-actin PCR products were 153, 214, 124, 153 and $128 \mathrm{bp}$, respectively. Primers were synthesized by Invitrogen.

Quantitative (real-time) PCR ( $q P C R$ ). Each DNA sample (2 $\mu 1,25 \mathrm{X}$ dilution) was suspended in $2 \mathrm{X}$ SYBR Premix Ex TaqT $^{\mathrm{TM}}$ (DRR041S; Takara,China) with 25 pmol of each sense and antisense primer, and double distilled water was added to a total volume of $25 \mu \mathrm{l}$. qPCR was performed using an ABI 7300 Real-Time PCR system (Applied Biosystems Foster City, CA, USA). The thermal profile was established according to the manufacturer's instructions. Briefly, this protocol consisted of enzyme activation at $95^{\circ} \mathrm{C}$ for $3 \mathrm{~min}$, followed by 45 cycles of denaturation at $95^{\circ} \mathrm{C}$ for $5 \mathrm{sec}$, and annealing and elongation at $52^{\circ} \mathrm{C}$ for $30 \mathrm{sec}$. For each run, a negative control tube without DNA was run along with the experimental samples. A 2-fold dilution series of the template was used in the qPCR assays. The HSPs and HSFl mRNA expression levels of all samples were normalized using the following formula: relative 


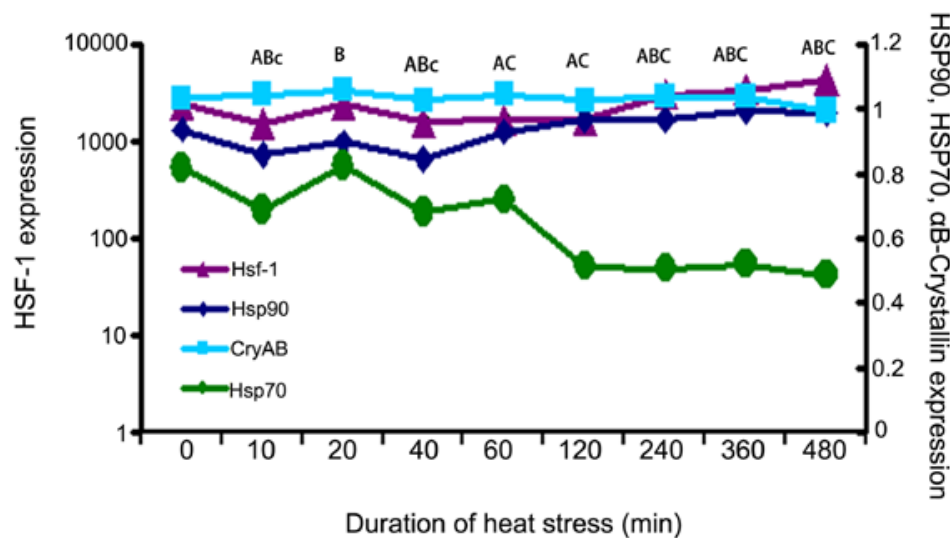

Figure 1. Heat shock proteins (HSPs) and heat shock factor (HSF1) protein expression in heat-stressed rat myocardial cells in vitro. HSPs and HSF1 levels were normalized to those of $\beta$-actin during $480 \mathrm{~min}$ of exposure to heat stress. HSF1 levels increased gradually and significantly from 240 min of exposure and onwards, and remained elevated after $480 \mathrm{~min}$ of heat stress. Compared with the control group, HSP90 expression decreased from 10 to $40 \mathrm{~min}$ of exposure to heat, and then increased from 60 to 480 min of heat stress. From 10 to $60 \mathrm{~min}$, the overall expression levels of HSP70 decreased compared with those of the control group, but not at 20 and $60 \mathrm{~min}$ of exposure. After $120 \mathrm{~min}$ of exposure, the level of HSP70 expression remained low until 480 min. There was no significant change in the crystallin, alpha B (CRYAB) levels during the $480 \mathrm{~min}$ of exposure to heat stress. The different letters each indicate a different factor as follows: Aa, HSF1; Bb, HSP90; Cc, SP70; Dd, CRYAB. Upper case letters (A, B and C) represent P<0.01; lower case letter (c) represents P<0.05. All the P-values were compared with the control group.

quantity of HSF1/HSP mRNA $=2^{-\Delta \Delta \mathrm{Ct}}$, where $\Delta \Delta \mathrm{Ct}=\left[\left(\mathrm{Ct}_{\mathrm{hs}} / \mathrm{hsps}\right.\right.$ mRNA - $\left.\mathrm{Ct}_{\text {-actin }} \mathrm{mRNA}\right)_{\text {test group }}-\left(\mathrm{Ct}_{\text {hsf/hsps }} \mathrm{mRNA}-\mathrm{Ct}_{\beta \text {-actin }}\right.$ mRNA) control group $_{\text {. }}$.

Analysis of HSFl and HSP interaction. We used the STRING (version 9.1) database (http://string-db.org/), which aims to provide a global perspective for as many organisms as feasible. The database scores and integrates known and predicted associations, resulting in comprehensive protein networks covering $>1,100$ organisms (31).

Statistical analysis. Statistical analysis of the differences between the experimental group and control group values was performed using one-way analysis of variance followed by the Duncan's multiple comparison test with SPSS version 20.0 software (IBM, Armonk, NY, USA). A value of $\mathrm{P}<0.05$ was considered to indicate a statistically significant difference when the experimental groups were compared with the controls. The values reported are the means $\pm \mathrm{SD}$. Three replicates were used for all experiments $(n=3)$.

\section{Results}

HSP and HSF1 expression under heat stress conditions. We detected and measured the expression levels of HSPs (HSP90, HSP70, CRYAB) and HSF1 by western blot analysis (Fig. 1). The results reaveled that HSF1 expression was significantly decreased $(\mathrm{P}<0.01)$ in response to heat stress for up to $120 \mathrm{~min}$ (Fig. 1). Following exposure to heat stress for $240 \mathrm{~min}$ or longer, the HSF1 expression levels gradually and significantly increased, and remained elevated following $480 \mathrm{~min}$ of exposure to heat stress (Fig. 1). Compared with the control group, HSP90 expression decreased following exposure of the cells to heat stress for 10 to $40 \mathrm{~min}$, and then increased from 60 to $480 \mathrm{~min}$ of exposure to heat stress, demonstrating an expression trend similar to that of HSF1 (Fig. 1). However, the epxression of HSP70 in the experimental group was decreased at 10 and $40 \mathrm{~min}$ of exposure to heat stress $(\mathrm{P}<0.05)$, and then further decreased significantly after $120 \mathrm{~min}$ of exposure to heat stress $(\mathrm{P}<0.01)$ until the time point of $480 \mathrm{~min}$ of exposure to heat stress (Fig. 1). The expression of HSP70 followed an opposite trend to that of HSF1. In contrast to the other HSPs, the CRYAB levels did not show any significant changes during the $480 \mathrm{~min}$ of exposure to heat stress (Fig. 1).

HSF1 and HSP mRNA mRNA levels under heat stress conditions. The mRNA levels of HSFl and HSPs are shown in Fig. 2. The mRNA expression of $\beta$-actin was not altered in response to heat stress (data not shown). The HSF1 mRNA levels increased rapidly and in a stepwise manner throughout the time course of exposure to heat stress and reached maximal levels (3-fold induction) after $480 \mathrm{~min}$ of exposure to heat stress (Fig. 2). The mRNA expression levels of $H S P 90$ increased after $10 \mathrm{~min}$ of exposure to heat stress and reached maximal levels after $240 \mathrm{~min}$ of exposure to heat stress ( $\mathrm{P}<0.01$; Fig. 2). All other time periods of exposure to heat stress resulted in significantly higher mRNA levels of HSP90 compared to the controls $(\mathrm{P}<0.01$; Fig. 2). The HSP70 mRNA levels increased significantly following $10 \mathrm{~min}$ of exposure to heat stress and continued to increase thereafter $(\mathrm{P}<0.01)$, reaching maximal levels at $360 \mathrm{~min}$ of exposure (Fig. 2). All the heat stress-associated genes were present at higher levels in the heat-stressed cells compared to the control group. The mRNA levels of $C R Y A B$ were also markedly increased in the heat-stressed myocardial cells ( $\mathrm{P}<0.01$; Fig. 2).

Association between HSF1 and HSPs. According to the rat data on the STRING database (version 9.1), HSF1 interacted with HSP70 and HSP90 (Fig. 3A). In the confidence view on the STRING database (Fig. 3B), a thicker line represented the association between HSF1 and HSP70 (indicating a stronger interacion) than that between HSF1 and HSP90. The interaction of HSF1 with HSP60 was not as strong as that between HSP70 and HSP90. However, there appeared to be no interaction between HSF1 and CRYAB. Under normal conditions in rats, HSF1 is not 


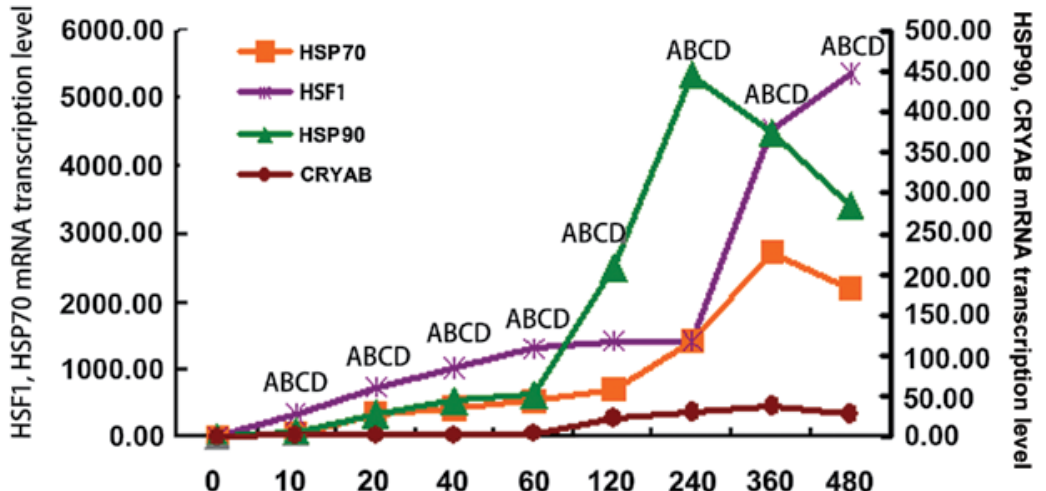

Duration of heat stress ( $\mathrm{min})$

Figure 2. mRNA expression of heat shock proteins $(H S P S)$ and heat shock factor $(H S F 1)$ in primary rat myocardial cells in vitro before and after exposure to heat stress. HSP and HSF1 mRNA levels were normalized to those of $\beta$-actin. HSF1 mRNA levels increased rapidly and in a stepwise manner throughout the time course and reached maximal levels (3-fold induction) after $360 \mathrm{~min}$ of exposure to heat stress. The mRNA level of $H S P 90$ increased after 10 min of exposure to heat stress, reaching the maximal level after $240 \mathrm{~min}$ of exposure. All other periods of exposure to heat stress resulted in significantly higher levels of $H S P 90 \mathrm{mRNA}$ than the controls. HSP70 mRNA increased significantly from 10 to $360 \mathrm{~min}$ of heat stress compared with the control group. Only the level at 480 min was slightly lower than 360 min of heat stress. The transcription levels of crystallin, alpha B (CRYAB) markedly increased in the heat-stressed myocardial cells. (Aa, HSF1; Bb, HSP90; Cc, HSP70; Dd, CRYAB. ABCD, P<0.01). The different letters each indicate a different factor as follows: A, HSF1; B, HSP90; C, HSP70; D, CRYAB. Upper case letters (A, B, C and D) represent $\mathrm{P}<0.01$. P-values were compared with the control group.

A
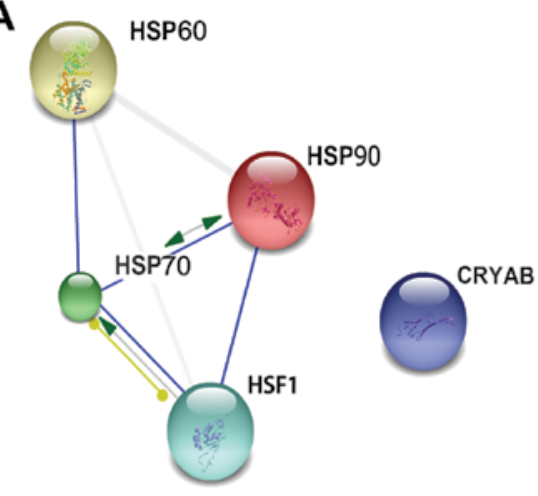

B

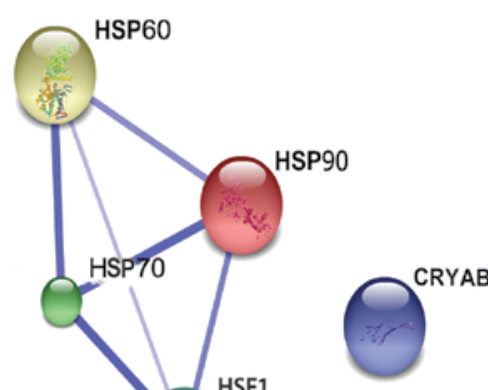

1.0

... from co-expression in other species (transferred):

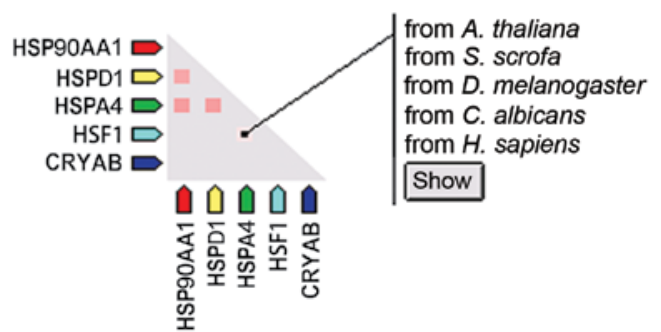

Figure 3. The association between heat shock factor 1 (HSF1) and heat shock proteins (HSPs) in rat myocardial cells analyzed with STRING (version 9.1) (A) HSF1 interacts with HSP90 and HSP70. HSF1 binds with HSP90 and HSP70 (blue line), HSF1 and HSP70 are co-expressed (yellow line), and HSF1 activates HSP70 (green line). (B) Thicker lines indicate a stronger interaction between two proteins. In rats, there is more evidence of an association between HSF1 and HSP70 than between HSF1 and HSP90. (C) Co-expression view showing that HSP70 and HSP60 are co-expressed with HSP90. However, in other species (e.g., Homo sapiens), HSF1 is co-expressed with HSP70.

co-expressed with any of the HSPs (Fig. 3C). However, there is evidence in other species that HSF1 is co-expressed with HSP70 (Homo sapiens). Of note, western blot analysis revealed that the protein expression levels of HSF1 followed a similar trend to those of HSP90 following exposure to heat stress, although the expression of HSP70 followed an opposite trend (Fig. 1). It has been suggested that HSP70 represses HSF1 during heat stress (12). Furthermore, a previous study indicated that HSF1 
A

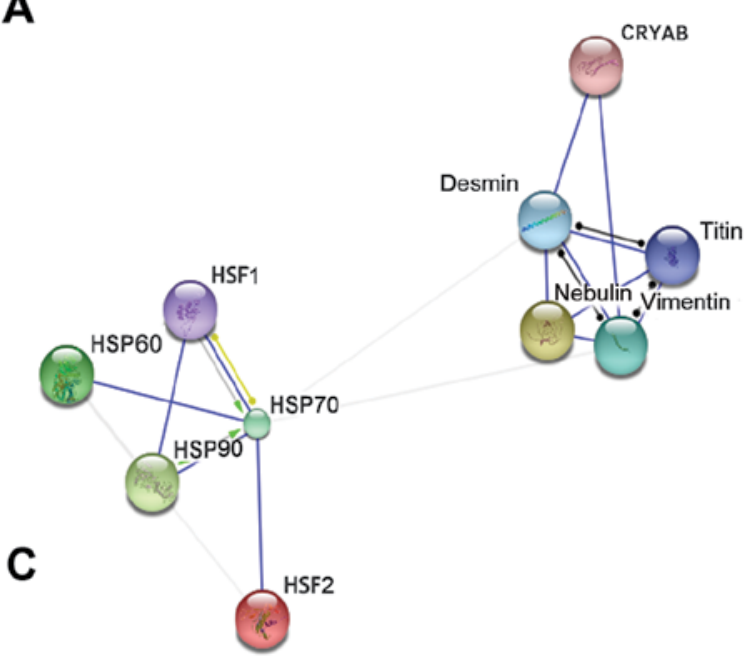

B

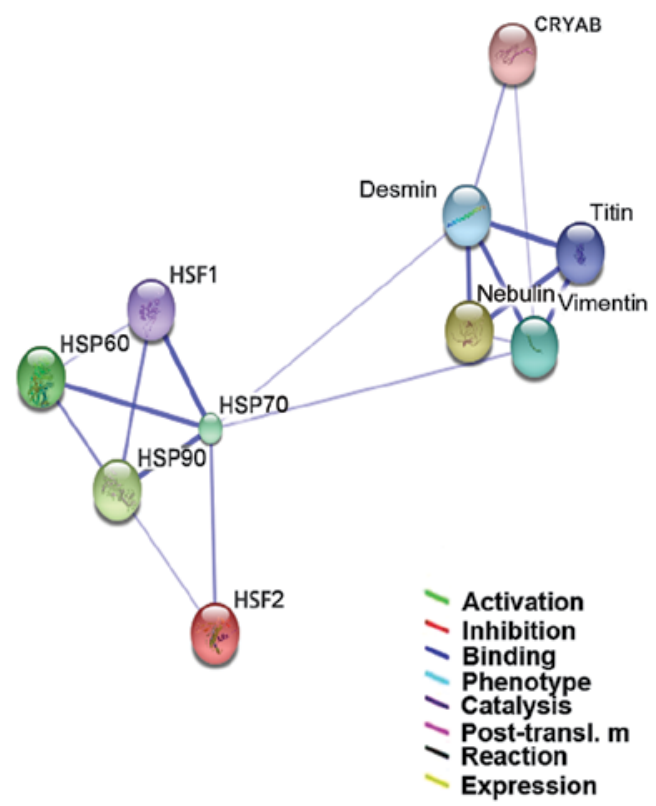

- co-expression association score 0.0 1.0 ... from co-expression in Rattus norvegicus:

... from co-expression in other species (transferred)

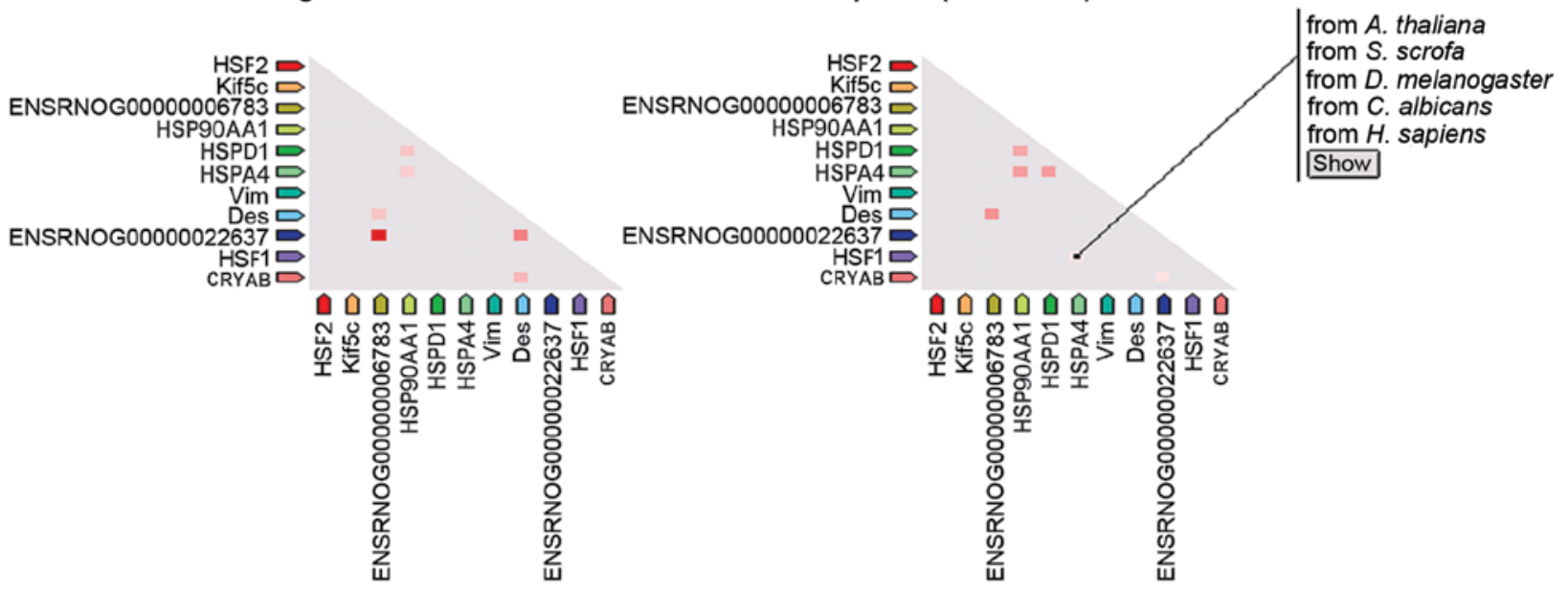

Figure 4. The interaction between heat shock factor (HSF)2 and heat shock proteins (HSPs) analyzed with STRING (version 9.1). (A) HSF2 interacts with HSP90 and HSP70. HSF2 binds with HSP90 and HSP70 (blue line). crystallin, alpha B (CRYAB) does not interact with HSF1 and HSF2; however, it binds with vimentin and desmin. (B) Thicker lines indicate more evidence of interaction between two proteins. In rats, there is more evidence of an association between HSF2 and HSP70 than between HSF2 and HSP90. (C) Co-expression view showing that CRYAB is co-expressed with desmin in rats.

is not the sole factor for HSP60 in rats [Buriro et al (36)]. Furthermore, data analysis confirmed that the expression levels of CRYAB were inconsistent with those of HSF1.

\section{Discussion}

The 'heat shock response' was first described in 1964 in Drosophila by Ritossa (32) and is characterized by the increased expression of a particular protein superfamily, HSPs. HSPs account for up to 2-3\% of the total cellular proteins at their baseline level, and their expression is strongly induced upon cellular stress $(33,34)$. This induction is mediated by a specific family of transcription factors, the HSFs. According to our results, the mRNA levels of $H S F 1$ and HSPs markedly increased from the onset of heat stress $(10 \mathrm{~min})$ compared with the control group. It has been demonstrated that heat stress induces $H S P$ gene folding (35), and our results are consistent with this finding. However, the HSF1 protein expression levels were not simulta- neously increased as were the $H S F 1$ mRNA levels during heat stress. These findings may be associated with the fact that a single HSF1 phosphorylation event has been shown to result in the rapid aggregation of HSF1 into a trimer that can bind to HSE and initiate the transcription process (36-39). The expression levels of HSP90 were also not consistent with its mRNA levels. The lack of a correlation between the mRNA levels of HSP 90 and the protein levels of HSP90 may be related to the complex post-transcriptional mechanisms that are involved in turning mRNAs into proteins (40). In the present study, the HSP70 protein levels decreased from 10 to $480 \mathrm{~min}$ of exposure to heat stress, except after $20 \mathrm{~min}$ of exposure to heat stress, which was also not consistent with its mRNA level. It has been reported that HSP70 reduces the infarct size in an in vivo transgenic mouse model of myocardial ischemia and reperfusion (41). HSP70 may interact with damaged proteins to play a protective role in rat primary myocardial cells. In this study, in contrast to its mRNA levels, the CRYAB protein expression levels were 
not significantly altered during $480 \mathrm{~min}$ of exposure to heat stress, which suggests that the protein expression was delayed or overtaxed due to the rapid consumption of CRYAB at the onset of heat stress. CRYAB is required for myocardial cell balance in response to stress (42). In our study, the mRNA levels of HSF1, $H S P 90, H S P 70$ and $C R Y A B$ increased significantly in primary neonatal rat myocardial cells in vitro following exposure to heat stress, and these factors may act as important markers in response to adverse environmental conditions in the heart (4).

Heat stress can contribute to protein misfolding, which in turn can trigger the stress response, leading to HSP expression (43). The transcription factor HSF1 induces HSP expression when the environmental temperature rises above the physiological range (44). HSF1 is inactive under normal conditions and is present in a heterocomplex with HSPs (45). Other studies, as well as ours, have observed different HSPs in normal and heat-stressed rat myocardial cells $(46,47)$. In the present study, HSF1 levels were increased after $120 \mathrm{~min}$ from $240 \mathrm{~min}$ of heat stress onwards. However, HSP70 expression tended to decrease following exposure to heat stress, particularly after $120 \mathrm{~min}$ of exposure to heat. STRING analysis determined that HSP70 was more closely connected with HSF1 than the other HSPs. Evidently, our findings indicate that HSF1 does not induce HSP70 expression in primary neonatal rat myocardial cells in vitro. It has been suggested that HSP70 is a major HSF1 repressor in human cells (48). Non-native proteins that accumulate under stress conditions may compete with HSF1 to bind to the chaperone heat shock (cognate) protein 70 (HSP/c70), and unbound HSF1 may homotrimerize and acquire transcriptional competence $(12,13)$. HSP70 prevents the in vitro conversion of HSF1 from a non-DNA-binding form to a DNA-binding form. It has also been suggested that HSP70 may play a regulatory role in HSF activation (12). The results of the present study indicated that HSP70 plays a repressive role, acting with HSF1 in rat heart cells in vitro. As shown in Fig. 3C, HSP70 is not co-expressed with other proteins in rats; however, the opposite is true in other species. In addition, it was observed that HSF2 interacts with HSP70 (Fig. 4A). Previous studies have confirmed that paternal HSF2 modifies endogenous HSP70.1 expression (49). However, the specific mechanisms through which HSF2 interacts with HSP70 in rat myocardial cells remain to be investigated.

HSP90 is a major soluble cellular protein and is most commonly located in the cytoplasm. It is considered a key factor at the crossroads between genetics and epigenetics, and it has been postulated that it is a capacitor for phenotypic variation and morphological evolution (50). The interaction between HSF1 and HSP90 appears to be dynamic (48). In the present study, HSP90 expression followed a similar trend to that of HSF1, suggesting that HSF1 plays a role in regulating HSP90. In human cells, a HSP90-containing HSF1 complex is formed during stress (48). Therefore, HSF1 may bind with HSP90 under stress conditions to play a protective role. In the present study (Fig. 4A), the interaction between HSF2 and HSP90 was very weak. Therefore, further confirmation of the mechanisms involved in this interaction is required.

In the highly crowded cellular environment, different chaperones follow distinct strategies to achieve the general goal of preventing protein misfolding and aggregation. As a member of the small HSP family, CRYAB acts as a molecular chaperone involved in increasing cellular tolerance to stress.
In vertebrates, CRYAB expression has been detected in the heart, eyes, lungs, liver and several other tissues under normal conditions $(51,52)$. CRYAB appears to be constitutively expressed in unstressed cells and is essential for maintaining cell homeostasis. It acts as a molecular chaperone to facilitate polypeptide transport, folding and assembly (51). In a previous study, the adenovirus-mediated transgenic overexpression of CRYAB revealed that in myocardial cells, the protein protects microtubules from acute ischemic damage (53). Whether HSFs can induce CRYAB expression in rat cardiac cells under stress conditions was one of the questions addressed in the present study. We found that the trend for CRYAB expression was not consistent with that of HSF1. Additionally, STRING analysis determined that CRYAB did not interact with either HSF1 or HSF2 (Figs. 3 and 4). It may interact with intermediate filaments to protect cytoskeletal organization in cardiomyocytes (24). In cardiomyocytes, CRYAB is localized within the I-band and M-line region of myofibrils, and appears to be involved in the organization of cytoskeletal structures (54). As shown in Fig. 4B, CRYAB binds with desmin and vimentin in rat cells in vitro, and may be co-expressed with desmin under certain conditions (Fig. 4C), suggesting that CRYAB may combine with desmin to play a protective role under stress conditions.

In conclusion, HSF1 is not the key HSF for all HSPs, particularly CRYAB, in rat myocardial cells in vitro. Moreover, HSF1 may play different roles in its interaction with HSPs in different species or cell types. STRING analysis confirmed our previous finding that HSF1 is not the sole factor for HSP60 (36).

\section{Acknowledgements}

This study was supported by grants from the National Key Basic Research Program of China (973 Program) (2014CB138502), the National Natural Science Foundation of China (31372403), the National Department Public Benefit Research Foundation (Agriculture) (201003060-11), the Priority Academic Program Development of Jiangsu Higher Education Institutions (PAPD), Graduate research and innovation projects in Jiangsu Province and the Sino-German Agricultural Cooperation Project of the Federal Ministry of Food, the Agriculture and Consumer Production, Berlin, Germany.

\section{References}

1. Gisolfi CV, Matthes RD, Kregel KC and Oppliger R: Splanchnic sympathetic nerve activity and circulating catecholamines in the hyperthermic rat. J Appl Physiol (1985) 70: 1821-1826, 1991.

2. Mirchandani HG, McDonald G, Hood IC and Fonseca C: Heat-related deaths in Philadelphia - 1993. Am J Forensic Med Pathol 17: 106-108, 1996.

3. Scheers-Masters JR, Schootman M and Thach BT: Heat stress and sudden infant death syndrome incidence: A United States population epidemiologic study. Pediatrics 113: e586-e592, 2004.

4. Yu J, Bao E, Yan J and Lei L: Expression and localization of Hsps in the heart and blood vessel of heat-stressed broilers. Cell Stress Chaperones 13: 327-335, 2008.

5. Zhang M, Lv Y, Yue Z, Islam A, Rehana B, Bao E and Hartung J: Effects of transportation on expression of Hsp90, Hsp70, Hsp27 and $\alpha \mathrm{B}$-crystallin in the pig stomach. Vet Rec 169: 312, 2011.

6. Li Z and Srivastava P: Heat-shock proteins. Curr Protoc Immunol (Appendix 1) 58: A.1T.1-A.1T.6, 2004.

7. Adhikari AS, Sridhar Rao K, Rangaraj N, Parnaik VK and Mohan Rao Ch: Heat stress-induced localization of small heat shock proteins in mouse myoblasts: Intranuclear lamin $\mathrm{A} / \mathrm{C}$ speckles as target for alphaB-crystallin and Hsp25. Exp Cell Res 299: 393-403, 2004. 
8. Borges JC and Ramos CH: Protein folding assisted by chaperones. Protein Pept Lett 12: 257-261, 2005.

9. Walter S and Buchner J: Molecular chaperones - cellular machines for protein folding. Angew Chem Int Ed Engl 41: 1098-1113, 2002.

10. Guisbert E, Yura T, Rhodius VA and Gross CA: Convergence of molecular, modeling, and systems approaches for an understanding of the Escherichia coli heat shock response. Microbiol Mol Biol Rev 72: 545-554, 2008.

11. Locke M and Tanguay RM: Diminished heat shock response in the aged myocardium. Cell Stress Chaperones 1: 251-260, 1996.

12. Abravaya K, Myers MP, Murphy SP and Morimoto RI: The human heat shock protein hsp70 interacts with HSF, the transcription factor that regulates heat shock gene expression. Genes Dev 6: 1153-1164, 1992.

13. Baler R, Zou $\mathbf{J}$ and Voellmy R: Evidence for a role of Hsp70 in the regulation of the heat shock response in mammalian cells Cell Stress Chaperones 1: 33-39, 1996.

14. Nathan DF, Vos MH and Lindquist S: In vivo functions of the Saccharomyces cerevisiae Hsp90 chaperone. Proc Natl Acad Sci USA 94: 12949-12956, 1997.

15. Jakob U, Meyer I, Bügl H, André S, Bardwell JC and Buchner J: Structural organization of procaryotic and eucaryotic Hsp90. Influence of divalent cations on structure and function. J Biol Chem 270: 14412-14419, 1995.

16. Young JC, Moarefi I and Hartl FU: Hsp90: A specialized but essential protein-folding tool. J Cell Biol 154: 267-273, 2001.

17. Nadeau K, Das A and Walsh CT: Hsp90 chaperonins possess ATPase activity and bind heat shock transcription factors and peptidyl prolyl isomerases. J Biol Chem 268: 1479-1487, 1993.

18. Arya R, Mallik M and Lakhotia SC: Heat shock genes - integrating cell survival and death. J Biosci 32: 595-610, 2007.

19. Kirchhoff SR, Gupta S and Knowlton AA: Cytosolic heat shock protein 60 , apoptosis, and myocardial injury. Circulation 105 : 2899-2904, 2002.

20. Veereshwarayya V, Kumar P, Rosen KM, Mestril R and Querfurth HW: Differential effects of mitochondrial heat shock protein 60 and related molecular chaperones to prevent intracellular $\beta$-amyloid-induced inhibition of complex IV and limit apoptosis. J Biol Chem 281: 29468-29478, 2006.

21. Malik ZA, Kott KS, Poe AJ, Kuo T, Chen L, Ferrara KW and Knowlton AA: Cardiac myocyte exosomes: Stability, HSP60, and proteomics. Am J Physiol Heart Circ Physiol 304: H954-H965, 2013.

22. Campanella C, Cappello F, Bucchieri F, et al: Hsp60 secretion and migration from cancer cells: A proposal for a multistage pathway. FASEB J 26: 521-526, 2012.

23. Fink AL: Chaperone-mediated protein folding. Physiol Rev 79: 425-449, 1999

24. Singh BN, Rao KS, Ramakrishna T, Rangaraj N and Rao ChM Association of $\alpha \mathrm{B}$-crystallin, a small heat shock protein, with actin: Role in modulating actin filament dynamics in vivo. J Mol Biol 366: 756-767, 2007.

25. Ashby RS, Megaw PL and Morgan IG: Changes in retinal alphaB-crystallin (cryab) RNA transcript levels during periods of altered ocular growth in chickens. Exp Eye Res 90: 238-243, 2010.

26. McCully JD, Lotz MM, Krukenkamp IB and Levitsky S: A brief period of retrograde hyperthermic perfusion enhances myocardial protection from global ischemia: Association with accumulation of Hsp 70 mRNA and protein. J Mol Cell Cardiol 28: 231-241, 1996.

27. Morimoto RI, Sarge KD and Abravaya K: Transcriptional regulation of heat shock genes. A paradigm for inducible genomic responses. J Biol Chem 267: 21987-21990, 1992.

28. Wu C: Heat shock transcription factors: Structure and regulation. Annu Rev Cell Dev Biol 11: 441-469, 1995.

29. Westerheide SD, Raynes R, Powell C, Xue B and Uversky VN: HSF transcription factor family, heat shock response, and protein intrinsic disorder. Curr Protein Pept Sci 13: 86-103, 2012.

30. Wu AM, Amdams LG and Pugh R: Immunochemical and partial chemical characterization of fractions of membrane-bound smooth lipopolysaccharide-protein complex from Brucella abortus. Mol Cell Biochem 75: 93-102, 1987.

31. Franceschini A, Szklarczyk D, Frankild S, et al: STRING v9.1: Protein-protein interaction networks, with increased coverage and integration. Nucleic Acids Res 41: D808-D815, 2013.

32. Ritossa FM: Experimental activation of specific loci in polytene chromosomes of Drosophila. Exp Cell Res 35: 601-607, 1964.

33. Ashburner $\mathrm{M}$ and Bonner JJ: The induction of gene activity in drosophilia by heat shock. Cell 17: 241-254, 1979.
34. Spradling A, Pardue ML and Penman S: Messenger RNA in heat-shocked Drosophila cells. J Mol Biol 109: 559-587, 1977.

35. Trinklein ND, Chen WC, Kingston RE and Myers RM: Transcriptional regulation and binding of heat shock factor 1 and heat shock factor 2 to 32 human heat shock genes during thermal stress and differentiation. Cell Stress Chaperones 9: 21-28, 2004.

36. Buriro R, Lv YJ, Ali I, Tang S, Liu ZJ, Zhang M, Adem A, Hartung J and Bao ED: Temporal variations of Hsp60 and HSF-1 in primary rat myocardial cells in vitro under heat stress. Genet Mol Res 12: 3003-3016, 2013.

37. Colett MS, Larson R, Gold C, Strick D, Anderson DK and Purchio AF: Molecular cloning and nucleotide sequence of the pestivirus bovine viral diarrhea virus. Virology 165: 191-199, 1988.

38. Cotto C, Berille J, Souquet PJ, Riou R, Croisile B, Turjman F, Giroux B, Brune J and Trillet-Lenoir V: A phase II trial of fotemustine and cisplatin in central nervous system metastases from non-small cell lung cancer. Eur J Cancer 32A: 69-71, 1996.

39. Sorger PK: Heat shock factor and the heat shock response. Cell 65: 363-366, 1991.

40. Islam A, Lv YJ, Abdelnasir A, et al: The role of Hsp90 $\alpha$ in heat-induced apoptosis and cell damage in primary myocardial cell cultures of neonatal rats. Genet Mol Res 12: 6080-6091, 2013.

41. Hutter JJ, Mestril R, Tam EK, Sievers RE, Dillmann WH and Wolfe CL: Overexpression of heat shock protein 72 in transgenic mice decreases infarct size in vivo. Circulation 94: 1408-1411, 1996.

42. Tang S, Buriro R, Liu Z, Zhang M, Ali I, Adam A, Hartung J and Bao E: Localization and expression of Hsp27 and $\alpha \mathrm{B}$-crystallin in rat primary myocardial cells during heat stress in vitro. PLoS One 8: e69066, 2013.

43. Ananthan J, Goldberg AL and Voellmy R: Abnormal proteins serve as eukaryotic stress signals and trigger the activation of heat shock genes. Science 232: 522-524, 1986.

44. Tulapurkar ME, Asiegbu BE, Singh IS and Hasday JD: Hyperthermia in the febrile range induces HSP72 expression proportional to exposure temperature but not to HSF-1 DNA-binding activity in human lung epithelial A549 cells. Cell Stress Chaperones 14: 499-508, 2009.

45. Santoro MG: Heat shock factors and the control of the stress response. Biochem Pharmacol 59: 55-63, 2000.

46. Madrigano J, Mittleman MA, Baccarelli A, Goldberg R, Melly S, von Klot S and Schwartz J: Temperature, myocardial infarction, and mortality: effect modification by individual and area-level characteristics. Epidemiology 24: 439-446, 2013.

47. Locke M, Noble EG, Tanguay RM, Feild MR, Ianuzzo SE and Ianuzzo CD: Activation of heat-shock transcription factor in rat heart after heat shock and exercise. Am J Physiol 268: C1387-C1394, 1995.

48. Zou J, Guo Y, Guettouche T, Smith DF and Voellmy R: Repression of heat shock transcription factor HSF1 activation by HSP90 (HSP90 complex) that forms a stress-sensitive complex with HSF1. Cell 94: 471-480, 1998.

49. Le Masson F and Christians E: HSFs and regulation of Hsp70.1 (Hspalb) in oocytes and preimplantation embryos: New insights brought by transgenic and knockout mouse models. Cell Stress Chaperones 16: 275-285, 2011.

50. Erlejman AG, Lagadari M, Toneatto J, Piwien-Pilipuk G and Galigniana MD: Regulatory role of the 90-kDa-heat-shock protein (Hsp90) and associated factors on gene expression. Biochim Biophys Acta 1839: 71-87, 2014.

51. Acunzo J, Katsogiannou M and Rocchi P: Small heat shock proteins HSP27 (HspB1), $\alpha \mathrm{B}$-crystallin (HspB5) and HSP22 (HspB8) as regulators of cell death. Int J Biochem Cell Biol 44: 1622-1631, 2012.

52. Garrido C, Paul C, Seigneuric R and Kampinga HH: The small heat shock proteins family: The long forgotten chaperones. Int J Biochem Cell Biol 44: 1588-1592, 2012.

53. Ray PS, Martin JL, Swanson EA, Otani H, Dillmann WH and Das DK: Transgene overexpression of alphaB crystallin confers simultaneous protection against cardiomyocyte apoptosis and necrosis during myocardial ischemia and reperfusion. FASEB J 15: 393-402, 2001.

54. Haskin CL, Athanasiou KA, Klebe R and Cameron IL: A heat-shock-like response with cytoskeletal disruption occurs following hydrostatic pressure in MG-63 osteosarcoma cells. Biochem Cell Biol 71: 361-371, 1993. 